\title{
Comparison Of The 25oh-vitamin D Levels Between Old Patients Hospitalized For Sars-cov-2 Pneumonia And Patients With Other Acute Illnesses: A Retrospective Case-control Study.
}

\section{Luca Tomisti ( $\nabla$ lucatomisti@gmail.com )}

Department of Internal Medicine, Apuane General Hospital, North-West Tuscany Healthcare District, 54100, Massa-Carrara, Italy

Nicolò Pulizzi

Department of Internal Medicine, Apuane General Hospital, North-West Tuscany Healthcare District, 54100, Massa-Carrara, Italy

\section{Pia Clara Pafundi}

Department of Advanced Medical and Surgical Sciences, University of Campania Luigi Vanvitelli, Naples, Italy

\section{Domenico Macaro}

Department of Internal Medicine, Apuane General Hospital, North-West Tuscany Healthcare District, 54100, Massa-Carrara, Italy

\section{Liliana Villari}

Pneumology Unit, Apuane General Hospital, North-West Tuscany Healthcare District, 54100, Massa-

Carrara, Italy

\section{Manuela Latorre}

Pneumology Unit, Apuane General Hospital, North-West Tuscany Healthcare District, 54100, MassaCarrara, Italy.

\section{Manuela Pogliaghi}

Infectious Disease Unit, Apuane General Hospital, North-West Tuscany Healthcare District, 54100, Massa-Carrara, Italy

\section{Mirco Lenzi}

Infectious Disease Unit, Apuane General Hospital, North-West Tuscany Healthcare District, 54100, Massa-Carrara, Italy

\section{Veronica Lenzi}

Department of Internal Medicine, Apuane General Hospital, North-West Tuscany Healthcare District, 54100, Massa-Carrara, Italy.

\section{Giulia Parri}

Pneumology Unit, Apuane General Hospital, North-West Tuscany Healthcare District, 54100, MassaCarrara, Italy. 


\section{Valentina Conti}

Pneumology Unit, Apuane General Hospital, North-West Tuscany Healthcare District, 54100, MassaCarrara, Italy.

\section{Paola Pacetti}

Oncology Unit, Apuane General Hospital, North-West Tuscany Healthcare District, 54100, MassaCarrara, Italy.

\section{Guido Bianchini}

Department of Internal Medicine, Apuane General Hospital, North-West Tuscany Healthcare District, 54100, Massa-Carrara, Italy.

\section{Alessandro Pampana}

Department of Internal Medicine, Apuane General Hospital, North-West Tuscany Healthcare District, 54100, Massa-Carrara, Italy.

\section{Research Article}

Keywords: COVID19, SARS-COV-2, 250H-vitamin D, pneumonia

Posted Date: October 23rd, 2020

DOI: https://doi.org/10.21203/rs.3.rs-96491/v1

License: (c) (1) This work is licensed under a Creative Commons Attribution 4.0 International License. Read Full License 
COMPARISON OF THE 25OH-VITAMIN D LEVELS BETWEEN OLD PATIENTS HOSPITALIZED FOR SARS-COV-2 PNEUMONIA AND PATIENTS WITH OTHER ACUTE ILLNESSES: A RETROSPECTIVE CASE-CONTROL STUDY.

Luca Tomisti ${ }^{1}$, Nicolò Pulizzi ${ }^{1}$, Pia Clara Pafundi² ${ }^{2}$ Domenico Macaro ${ }^{1}$, Liliana Villari ${ }^{3}$, Manuela Latorre $^{3}$, Manuela Pogliaghi ${ }^{4}$, Mirco Lenzi ${ }^{4}$, Veronica Lenzi ${ }^{1}$, Giulia Parri ${ }^{3}$, Valentina Conti ${ }^{3}$, Paola Pacetti $^{5}$, Guido Bianchini ${ }^{1}$, and Alessandro Pampana ${ }^{1}$

1Department of Internal Medicine, Apuane General Hospital, North-West Tuscany Healthcare District, 54100, Massa-Carrara, Italy.

2Department of Advanced Medical and Surgical Sciences, University of Campania Luigi Vanvitelli, Naples, Italy

3Pneumology Unit, Apuane General Hospital, North-West Tuscany Healthcare District, 54100, Massa-Carrara, Italy.

4Infectious Disease Unit, Apuane General Hospital, North-West Tuscany Healthcare District, 54100, Massa-Carrara, Italy.

${ }^{5}$ Oncology Unit, Apuane General Hospital, North-West Tuscany Healthcare District, 54100, MassaCarrara, Italy.

Corresponding Author: Luca Tomisti, MD, Department of Internal Medicine, Apuane General Hospital, North-West Tuscany Healthcare District, Via Enrico Mattei, 21, 54100 Massa (MS) Italy; phone +39-349-2637596; Fax: +39-(0)50-997249; e-mail: lucatomisti@gmail.com luca.tomisti@uslnordovest.toscana.it.

Short Title: 25OH-Vitamin D in SARS-COV-2 pneumonia.

Key Words: COVID19, SARS-COV-2, 25OH-vitamin D, pneumonia, 


\section{ABSTRACT}

PURPOSE. To assess and compare the serum 25OH-vitamin D levels in three cohorts of patients hospitalized due to acute illness, either related or not to a SARS-COV-2 infection. To investigate, in the patients group with SARS-COV-2 pneumonia, the possible relationship between the serum vitamin D levels and both disease severity and mortality risk.

METHODS. This is a retrospective case-control study. Serum 25OH-vitamin D levels were compared between patients with SARS-COV-2 pneumonia (COVID-19 group, 52 patients), and two control groups, including patients with pneumonia not related to SARS-CoV-2 (NO COVID1 group, 52 patients) and patients with a non-respiratory acute disease (NO COVID2 group, 52 patients).

RESULTS. No differences were found in the serum $25 \mathrm{OH}-$ Vitamin D levels among the three groups. In the COVID-19 group, serum 25 OH-Vitamin D levels did not show significant association with mortality risk $(\mathrm{p}=0.12)$, Intensive Care Unit admission risk $(\mathrm{p}=0.36)$, inpatients duration $(\mathrm{p}=0.40)$ and remission time $(\mathrm{p}=0.33)$. Similar results were found for parameters estimating the disease severity, such as basal PO2/FiO2 $(p=0.77)$, worse PO2/FiO2 ( $p=0.41)$, basal D-dimer $(p=0.46)$ and basal LDH $(\mathrm{p}=0.52)$.

CONCLUSIONS. Our data do not show lower 25OH-vitamin D levels in the patients with SARSCOV-2 pneumonia compared to patients hospitalized for other acute illnesses. In the COVID-19 group the $25 \mathrm{OH}$-vitamin D levels did not show significant correlation with a worse outcome. 


\section{INTRODUCTION}

In January 2020, a new coronavirus named SARS-CoV-2 was identified from a nasal swab specimen in the Hubei Region, China. SARS-CoV-2 is an enveloped RNA virus, mainly affecting the respiratory tract. The virus can trigger a wide range of responses, from complete lack of symptoms to atypical pneumonia with cytokine storm and acute respiratory distress syndrome (ARDS). The World Health Organization (WHO) named this clinical syndrome COVID-19. In March 2020, COVID-19 has been declared a global pandemic.

Vitamin D is normally produced in the skin after exposure to adequate levels of UVB-light and it is mainly involved in bone metabolism.

Several studies have explored the potential role of vitamin D supplementation both in protecting against respiratory infection [1-3] and in reducing the rate of asthma exacerbations [4]. On the other hand, the role of vitamin D supplementation in the reduction of the risk of flu is controversial [5-7]. Several mechanisms have been proposed in order to explain the protective effect of vitamin D against microbial infection: 1) it maintains tight junctions, gap junctions and adherent junctions, thus creating a physical barrier effect [8];2) it enhances cellular innate immunity, by inducing antimicrobial peptides [9], reducing the expression of pro-inflammatory cytokines and increasing the expression of anti-inflammatory cytokines by macrophages [10]; 3) it modulates the adaptive immunity, by suppressing responses mediated by the type $1 \mathrm{~T}$ helper (Th1) cell [11], promoting cytokine production by the type $2 \mathrm{~T}$ helper (Th2) cells [12] and inducing T regulatory cells [13].

On these bases, some recent editorials have proposed a potential protective role of vitamin D against COVID-19 infection risk and, consequently, a relationship between vitamin D deficiency and severity of infection $[7,14,15]$.

We thus compared the $25 \mathrm{OH}$-vitamin D levels of a cohort of patients hospitalized for COVID-19 pneumonia with two cohorts of patients referred to our hospital in the same study period because of pneumonia not related to COVID19 and because of a non-respiratory related acute disease, respec- 
tively. We further assessed, in the COVID-19 group, the potential relationship between serum vitamin D levels and either disease severity or mortality. 


\section{MATERIALS AND METHODS}

\section{STUDY DESIGN AND GROUPS DEFINITION}

This is a retrospective case-control study including patients consecutively admitted to Apuane General Hospital of Massa, Italy from March 15, 2020 to May 11, 2020. All anthropometric, demographic and clinical data were collected from electronic records, and revised from a single operator. The COVID-19 group included patients affected by a SARS-Cov-2 related pneumonia. SARS-CoV2 infection was confirmed by the positivity of an oropharyngeal and/or nasopharyngeal swab. Inclusion criteria included the presence of radiological findings of pneumonia (detected at CT scan) and performance of a $25 \mathrm{OH}$-vitamin D serum sample during hospitalization. The case-control ratio was $1: 1: 1$

Non-COVID groups included patients hospitalized during the same study period, with at least two consecutive negative oropharyngeal and/or nasopharyngeal swabs within 24-hours of each other and a serum $25 \mathrm{OH}$-vitamin D determination during hospitalization.

The first control group included consecutive patients referred to our hospital due to a lobar or interstitial pneumonia (Non-COVID 1 group), whilst the second group included consecutive patients referred to our hospital for other non-respiratory acute diseases (Non-COVID 2 group). In particular, Non-COVID 2 group was characterized as follows: nine patients with sepsis, 4 with hematological disease, 13 with gastroenterological disease, 9 with heart failure, 7 with malignancies, and 19 with neurologic disease.

Each patient gave her/his written informed consent, at the first clinical visit at our Department to the use of clinical data for research purpose. The study was approved by the Internal Review Board of our Department and is in accordance with 1976 Declaration of Helsinki and its later amendments.

\section{ENDPOINTS AND DEFINITIONS}


The primary endpoint was to compare the serum $25 \mathrm{OH}$-vitamin D levels between COVID-19 group and the two control groups (Non-COVID 1 group and Non-COVID 2 group). The secondary endpoint was to investigate in the COVID-19 group the possible relationship between the serum vitamin D levels and both disease severity and in-hospital mortality.

Basal D-dimer and basal LDH were collected during the first 24 hours after hospital admission. We collected basal $\mathrm{PO} 2 / \mathrm{FiO} 2$ (ratio of arterial oxygen partial pressure to fractional inspired oxygen) during the first 24 hours after hospital admission. We defined worse $\mathrm{P} / \mathrm{F}$ the lowest $\mathrm{P} / \mathrm{F}$ value detected at any time during the patient hospitalization.

Inpatient duration was defined as the time between admission and discharge; remission time was defined as the time between admission and clinical remission, i.e. absence of fever for at least 48 hours, without relapse, and resolution of respiratory failure.

Respiratory failure was considered resolved with values of $\mathrm{PO} 2 / \mathrm{FiO} 2$ ratio $>300$ or $\mathrm{SpO} 2>94 \%$, without oxygen supplementation (90\% for patients with chronic respiratory failure).

\author{
ASSAYS \\ 25OH-Vitamin D (LIAISON 25OH-Vitamin D TOTAL Assay, DiaSorin, Saluggia, Vercelli, IT). \\ PTH (LIAISON 1-84 PTH, DiaSorin, Saluggia, Vercelli, IT). Calcium (Calcium [Arsenazo], Beck- \\ man Coulter, Brea, California, US). D-Dimer (HemosIL D-Dimer HS500, Werfen, L'Hospitalet de \\ Llobregat, Barcelona, ESP). LDH (Lactate Dehydrogenase, LD, Beckman Coulter, Brea, California, \\ US).
}

\title{
STATISTICAL ANALYSIS
}

Categorical variables were expressed as number and percentages. Continuous variables were expressed as median and interquartile range [IQR] or mean and standard deviation (SD), according to their distribution, appropriately assessed by the Shapiro-Wilk test. 
Between-groups differences were assessed by the Chi-Square $\left(\mathrm{X}^{2}\right)$ test in the case of categorical variables, whereas continuous variables were analyzed either by the one-way analysis of variance test (ANOVA) or by the Kruskal-Wallis test, as appropriate.

Univariate linear regressions were instead performed to test whether Basal D dimer and LDH were correlated with serum $25 \mathrm{OHD}$ levels. Logistic regressions were finally performed to assess the potential relationship between serum $25 \mathrm{OH}$-vitamin D levels and the outcomes.

A p-value $<0.05$ was considered as statistically significant. SPSS Software, Version 24 (IBM, Armonk, New York) performed all analyses. 


\section{RESULTS}

Fifty-two COVID-19 patients were finally included in the COVID-19 group, almost equally distributed for sex (25 males vs. 27 females), with a median age of 84 [IQR 78-89] years. All patients were hospitalized for a median time of 15 [IQR 10-23] days and treated with medical therapy, according to the recommendations valid at that time. Sixteen patients $(30.8 \%)$ died. Clinical and biochemical features of COVID-19 group are shown in Table 1.

Thirty-nine patients (75\%) had hypertension, 20 (61.5\%) other cardiovascular diseases, $16(30.8 \%)$ diabetes, 8 (15.4\%) COPD and $5(9.6 \%)$ an active cancer (Table 2).

The COVID-19 group was compared with two control groups (Non-COVID 1 and Non-COVID 2 group, respectively). The three groups did not differ significantly for age, sex and basal serum creatinine. As well, the three groups did not show significant differences in the $25 \mathrm{OH}-$ Vitamin $\mathrm{D}$ serum levels, in the serum PTH and in the serum calcium corrected for albumin (Table 3).

In the univariate analysis, the $25 \mathrm{OH}-$ Vitamin D levels of the COVID-19 group were not significantly associated with mortality risk $(\mathrm{p}=0.12)$, Intensive Care Unit (ICU) admission risk $(\mathrm{p}=0.36)$, duration of hospitalization $(p=0.40)$ and remission time $(p=0.33)$. No significant association were found either for markers of disease severity, such as basal $\mathrm{PO} 2 / \mathrm{FiO} 2$ ratio $(\mathrm{p}=0.70)$, lowest $\mathrm{P} / \mathrm{F}$ ratio during hospitalization $(p=0.41)$, basal D dimer $(p=0.34)$ and basal LDH $(p=0.54)$, as reported in Table 4.

Ten patients (20\%) in the COVID-19 group required ICU admission and received ventilatory support for a median time of 8 [IQR 6-9] days. Figure 1 shows that serum 25OH-vitamin D levels did not significantly differ between patients requiring or not ICU admission (15.8 [IQR 10.1-36.2] ng/ $\mathrm{ml}$ vs $13.5[7.2-28.7] \mathrm{ng} / \mathrm{ml} ; \mathrm{p}=0.49)$.

Sixteen out of 52 COVID-19 group patients (30.8\%) died. As expected, patients admitted to ICU and underwent ventilatory support showed a higher mortality rate comparing to the patients man- 
aged in a medical ward $(60 \%$ vs $23.8 \%, \mathrm{p}=0.03)$. Figure 2 shows that serum $25 \mathrm{OH}$-vitamin D values were not significantly different between the dead patients and survivors (22.9 [IQR 9.2-31.1] $\mathrm{ng} / \mathrm{ml}$ vs. $11.8[7.1-26.8] \mathrm{ng} / \mathrm{ml} ; \mathrm{p}=0.14)$ despite the presence of a trend to be higher in the dead patients. 


\section{DISCUSSION}

It has been widely reported that Vitamin D deficiency may represent a risk factor for respiratory tract infections. In fact, vitamin D affects the expression of many genes linked to airway infections, as well as the normal function of the immune system. Nevertheless, the role of vitamin D supplementation remains controversial. A meta-analysis by Martineau et al concluded that Vitamin D supplementation reduces the risk of acute respiratory infection [3]. Likewise Joliff et al concluded that vitamin D supplementation reduced the rate of asthma exacerbations, requiring treatment with systemic corticosteroids [4].

A recent narrative review reported that several countries in the Northern Hemisphere had a high COVID-19 mortality, even after adjustment for age, thus suggesting that the association between COVID-19 mortality and latitude may be related to an effect of ultraviolet light and therefore to the vitamin D status [14]. Similarly, a European study showed a negative correlation between mean country vitamin D levels and number of COVID-19 cases and between mean vitamin D levels and COVID-19 mortality risk [16].

In keeping with these observations, a potential role of the vitamin D on COVID-19 infection risk, hospitalization and mortality has been recently discussed, up to suggesting a widespread supplementation with vitamin D despite the absence of randomized control trials $[7,17,18]$.

In our study, we investigated the vitamin D status in a cohort of patients referred to our hospital due to a SARS-CoV-2 related pneumonia (COVID-19 group). Our cohort included a large number of elderly patients (median age 84 years), which may explain the higher mortality rate $(30.8 \%)$, consistent with the national mortality rates reported for patients older than 80 years (National Healt Institute, ISS, updated on 8th September, 2020).

It is well known that ill patients could show lower vitamin D levels in comparison with healthy controls, as vitamin D binding protein and albumin concentrations are remarkably reduced during acute illness and inflammatory activation $[19,20]$. Therefore, we compared the vitamin D status of 
of the COVID-19 patients group with a control group composed by patients referred to our hospital, in the same period, because of a pneumonia not related to SARS-CoV-2 infection, failing to find significant differences.

As respiratory acute infections may be considered an independent risk factor for a $25 \mathrm{OH}$-vitamin D insufficiency we further compared the vitamin D status of the COVID-19 group with a second control group composed by patients admitted for a non-respiratory acute illness. Even in this case, we did not find a significant difference in the serum vitamin D status.

Data of the literature are conflicting. A recent large UK Biobank study, after adjustment for confounding factors, did not show correlations between the serum vitamin D levels and the risk of COVID-19 infection [21]. On the contrary, other series reported a higher prevalence of $25 \mathrm{OH}$-vitamin D deficiency in patients with a COVID-19 positive test [22-24]. Of note, in these studies a significant timeframe between the serum Vitamin D sample collections and COVID-19 infection elapsed (up to 12 months), which may produce a bias, whereas all the three groups of our study collected the $25 \mathrm{OH}-$ Vitamin D blood sample on admission to hospital. Furthermore, most of these studies were conducted on patients significantly younger than those included in our study, where 45 out of 52 patients $(86 \%)$ were older than 70 years and median age was 84 .

In addition, in our cohort of patients affected by COVID-19 pneumonia no correlations were found among the serum vitamin D levels and the mortality risk, the ICU admission risk, the remission time and the duration of hospitalization, as well as with the parameters estimating the disease severity.

Even in this case, available data is inconsistent. A recent study, conducted in a geriatric cohort of patients, has associated vitamin D deficiency with a higher peak of D-dimer levels and a higher risk of needing ventilatory support. Nevertheless, neither mortality nor duration of hospitalization was associated with the vitamin D status [25]. Likewise, other small series reported a higher prevalence of $25 \mathrm{OH}-\mathrm{vitamin} \mathrm{D}$ deficiency in the patients with a worse outcome [26] or in those requiring ICU admission [27]

In conclusion, our results do not support the hypothesis of a specific role of the vitamin D deficiency in the risk of COVID-19 infection. In fact, no differences were found between the $25 \mathrm{OH}$-vitamin 
D levels of the patients affected by COVID-19 related pneumonia and those of other patients hospitalized because of other acute illnesses.

In addition, in the group of patients with COVID-19 related pneumonia, serum 25OH-vitamin D levels did not disclose significant association neither with the main outcome of COVID-19 infection (mortality and need of mechanical ventilation) nor with the parameters estimating the severity of disease.

Our study discloses several limitations, as first the retrospective design and the small sample size. However, to our knowledge, the is the first report comparing the vitamin D status of a cohort of patients with SARS-CoV-2 pneumonia to others cohorts of hospitalized patients affected by acute diseases. Further larger studies are needed to confirm these observations. 
COMPLIANCE WITH ETHICAL STANDARDS

Disclosure of potential conflicts of interest:The authors have no relevant financial or non-financial interests to disclose.

Research involving Human and/or Animals: This research study was conducted retrospectively from data obtained for clinical purposes. The study was approved by the Internal Review Board of our Department and is in accordance with 1976 Declaration of Helsinki and its later amendments. All the procedures being performed were part of the routine care.

Informed consent: Each patient gave her/his written informed consent, at the first clinical visit at our Department, to the use of clinical data for research purpose. The authors affirm that participants provided informed consent for publication. 


\section{ACKNOLEDGEMENTS}

Study Group Investigators: The COVID-19 team of Ospedale Apuane, Massa, Italy includes the following participants: Carlo Zannoni, MD, Marta Bongiorni, MD, Marco Morale, MD, Giuliana Andreani, MD, Lucia Tonarelli, MD, Fausto Ceccarelli, MD, Emily Piccotti, MD, Beatrice Rosaia, MD, Daniela Moruzzo, MD, Francesca Madesani, MD, Francesca Angelotti, MD, Dante Giumetti, MD, Franca Ponzanelli, MD, Luigi Di Palma, MD, Anna Porcu, MD, Sara Balestracci, MD, Alberto Rocchi, MD, Sergio Laganà, MD, Antonella Vincenti, MD.

I am grateful to Prof. Fausto Bogazzi, University of Pisa, for his continuous and unconditional support. 


\section{LEGEND TO FIGURE}

Figure 1. Comparison of the serum $25 \mathrm{OH}$-vitamin D levels between the patients requiring mechanical ventilatory support in an Intensive Care Unit (ICU) $(n=10)$ and the patients managed in a medical ward $(n=42)$.

Figure 2. Comparison of the serum $25 \mathrm{OH}$-vitamin D levels between the subgroup of patients died during the hospital stay $(\mathrm{n}=16)$ and the subgroup of survivors $(\mathrm{n}=36)$. 


\section{BIBLIOGRAPHY}

1. Bergman P, Lindh AU, Bjorkhem-Bergman L, Lindh JD (2013) Vitamin D and Respiratory Tract Infections: A Systematic Review and Meta-Analysis of Randomized Controlled Trials. PLoS One 8 (6):e65835. doi:10.1371/journal.pone.0065835

2. Jolliffe DA, Griffiths CJ, Martineau AR (2013) Vitamin D in the prevention of acute respiratory infection: systematic review of clinical studies. J Steroid Biochem Mol Biol 136:321-329. doi:10.1016/j.jsbmb.2012.11.017

3. Martineau AR, Jolliffe DA, Hooper RL, Greenberg L, Aloia JF, Bergman P, Dubnov-Raz G, Esposito S, Ganmaa D, Ginde AA, Goodall EC, Grant CC, Griffiths CJ, Janssens W, Laaksi I, Manaseki-Holland S, Mauger D, Murdoch DR, Neale R, Rees JR, Simpson S, Jr., Stelmach I, Kumar GT, Urashima M, Camargo CA, Jr. (2017) Vitamin D supplementation to prevent acute respiratory tract infections: systematic review and meta-analysis of individual participant data. BMJ 356:i6583. doi:10.1136/bmj.i6583

4. Jolliffe DA, Greenberg L, Hooper RL, Griffiths CJ, Camargo CA, Jr., Kerley CP, Jensen ME, Mauger D, Stelmach I, Urashima M, Martineau AR (2017) Vitamin D supplementation to prevent asthma exacerbations: a systematic review and meta-analysis of individual participant data. Lancet Respir Med 5 (11):881-890. doi:10.1016/S2213-2600(17)30306-5

5. Urashima M, Mezawa H, Noya M, Camargo CA, Jr. (2014) Effects of vitamin D supplements on influenza A illness during the 2009 H1N1 pandemic: a randomized controlled trial. Food Funct 5 (9):2365-2370. doi:10.1039/c4fo00371c

6. Gruber-Bzura BM (2018) Vitamin D and Influenza-Prevention or Therapy? Int J Mol Sci 19 (8). doi:10.3390/ijms19082419

7. Grant WB, Lahore H, McDonnell SL, Baggerly CA, French CB, Aliano JL, Bhattoa HP (2020) Evidence that Vitamin D Supplementation Could Reduce Risk of Influenza and COVID-19 Infections and Deaths. Nutrients 12 (4). doi:10.3390/nu12040988 
8. Schwalfenberg GK (2011) A review of the critical role of vitamin D in the functioning of the immune system and the clinical implications of vitamin D deficiency. Mol Nutr Food Res 55 (1):96-108. doi:10.1002/mnfr.201000174

9. Laaksi I (2012) Vitamin D and respiratory infection in adults. Proc Nutr Soc 71 (1):90-97. doi:10.1017/S0029665111003351

10. Gombart AF, Pierre A, Maggini S (2020) A Review of Micronutrients and the Immune SystemWorking in Harmony to Reduce the Risk of Infection. Nutrients 12 (1). doi:10.3390/nu12010236

11. Lemire JM, Adams JS, Kermani-Arab V, Bakke AC, Sakai R, Jordan SC (1985) 1,25-Dihydroxyvitamin D3 suppresses human T helper/inducer lymphocyte activity in vitro. J Immunol 134 (5):3032-3035

12. Cantorna MT, Snyder L, Lin YD, Yang L (2015) Vitamin D and 1,25(OH)2D regulation of T cells. Nutrients 7 (4):3011-3021. doi:10.3390/nu7043011

13. Jeffery LE, Burke F, Mura M, Zheng Y, Qureshi OS, Hewison M, Walker LS, Lammas DA, Raza K, Sansom DM (2009) 1,25-Dihydroxyvitamin D3 and IL-2 combine to inhibit T cell production of inflammatory cytokines and promote development of regulatory T cells expressing CTLA-4 and FoxP3. J Immunol 183 (9):5458-5467. doi:10.4049/jimmunol.0803217

14. Rhodes JM, Subramanian S, Laird E, Griffin G, Kenny RA (2020) Perspective: Vitamin D deficiency and COVID-19 severity - plausibly linked by latitude, ethnicity, impacts on cytokines, ACE2 and thrombosis. J Intern Med. doi:10.1111/joim.13149

15. Trovas G, Tournis S (2020) Vitamin D and COVID-19. Hormones (Athens). doi:10.1007/ s42000-020-00231-9

16. Ilie PC, Stefanescu S, Smith L (2020) The role of vitamin D in the prevention of coronavirus disease 2019 infection and mortality. Aging Clin Exp Res 32 (7):1195-1198. doi:10.1007/ s40520-020-01570-8 
17. Jakovac H (2020) COVID-19 and vitamin D-Is there a link and an opportunity for intervention? Am J Physiol Endocrinol Metab 318 (5):E589. doi:10.1152/ajpendo.00138.2020

18. Weir EK, Thenappan T, Bhargava M, Chen Y (2020) Does vitamin D deficiency increase the severity of COVID-19? Clin Med (Lond) 20 (4):e107-e108. doi:10.7861/clinmed.2020-0301

19. Nonnecke BJ, McGill JL, Ridpath JF, Sacco RE, Lippolis JD, Reinhardt TA (2014) Acute phase response elicited by experimental bovine diarrhea virus (BVDV) infection is associated with decreased vitamin D and E status of vitamin-replete preruminant calves. J Dairy Sci 97 (9):5566-5579. doi:10.3168/jds.2014-8293

20. Silva MC, Furlanetto TW (2015) Does serum 25-hydroxyvitamin D decrease during acute-phase response? A systematic review. Nutr Res 35 (2):91-96. doi:10.1016/j.nutres.2014.12.008

21. Hastie CE, Mackay DF, Ho F, Celis-Morales CA, Katikireddi SV, Niedzwiedz CL, Jani BD, Welsh P, Mair FS, Gray SR, O'Donnell CA, Gill JM, Sattar N, Pell JP (2020) Vitamin D concentrations and COVID-19 infection in UK Biobank. Diabetes Metab Syndr 14 (4):561-565. doi:10.1016/ j.dsx.2020.04.050

22. Kaufman HW, Niles JK, Kroll MH, Bi C, Holick MF (2020) SARS-CoV-2 positivity rates associated with circulating 25-hydroxyvitamin D levels. PLoS One 15 (9):e0239252. doi:10.1371/journal.pone. 0239252

23. Meltzer DO, Best TJ, Zhang H, Vokes T, Arora V, Solway J (2020) Association of Vitamin D Status and Other Clinical Characteristics With COVID-19 Test Results. JAMA Netw Open 3 (9):e2019722. doi:10.1001/jamanetworkopen.2020.19722

24. Merzon E, Tworowski D, Gorohovski A, Vinker S, Golan Cohen A, Green I, Frenkel-Morgenstern M (2020) Low plasma 25(OH) vitamin D level is associated with increased risk of COVID-19 infection: an Israeli population-based study. FEBS J. doi:10.1111/febs.15495 
25. Baktash V, Hosack T, Patel N, Shah S, Kandiah P, Van den Abbeele K, Mandal AKJ, Missouris CG (2020) Vitamin D status and outcomes for hospitalised older patients with COVID-19. Postgrad Med J. doi:10.1136/postgradmedj-2020-138712

26. Carpagnano GE, Di Lecce V, Quaranta VN, Zito A, Buonamico E, Capozza E, Palumbo A, Di Gioia G, Valerio VN, Resta O (2020) Vitamin D deficiency as a predictor of poor prognosis in patients with acute respiratory failure due to COVID-19. J Endocrinol Invest. doi:10.1007/ s40618-020-01370-X

27. Panagiotou G, Tee SA, Ihsan Y, Athar W, Marchitelli G, Kelly D, Boot CS, Stock N, Macfarlane J, Martineau AR, Burns G, Quinton R (2020) Low serum 25-hydroxyvitamin D (25[OH]D) levels in patients hospitalized with COVID-19 are associated with greater disease severity. Clin Endocrinol (Oxf). doi:10.1111/cen.14276 
Figures

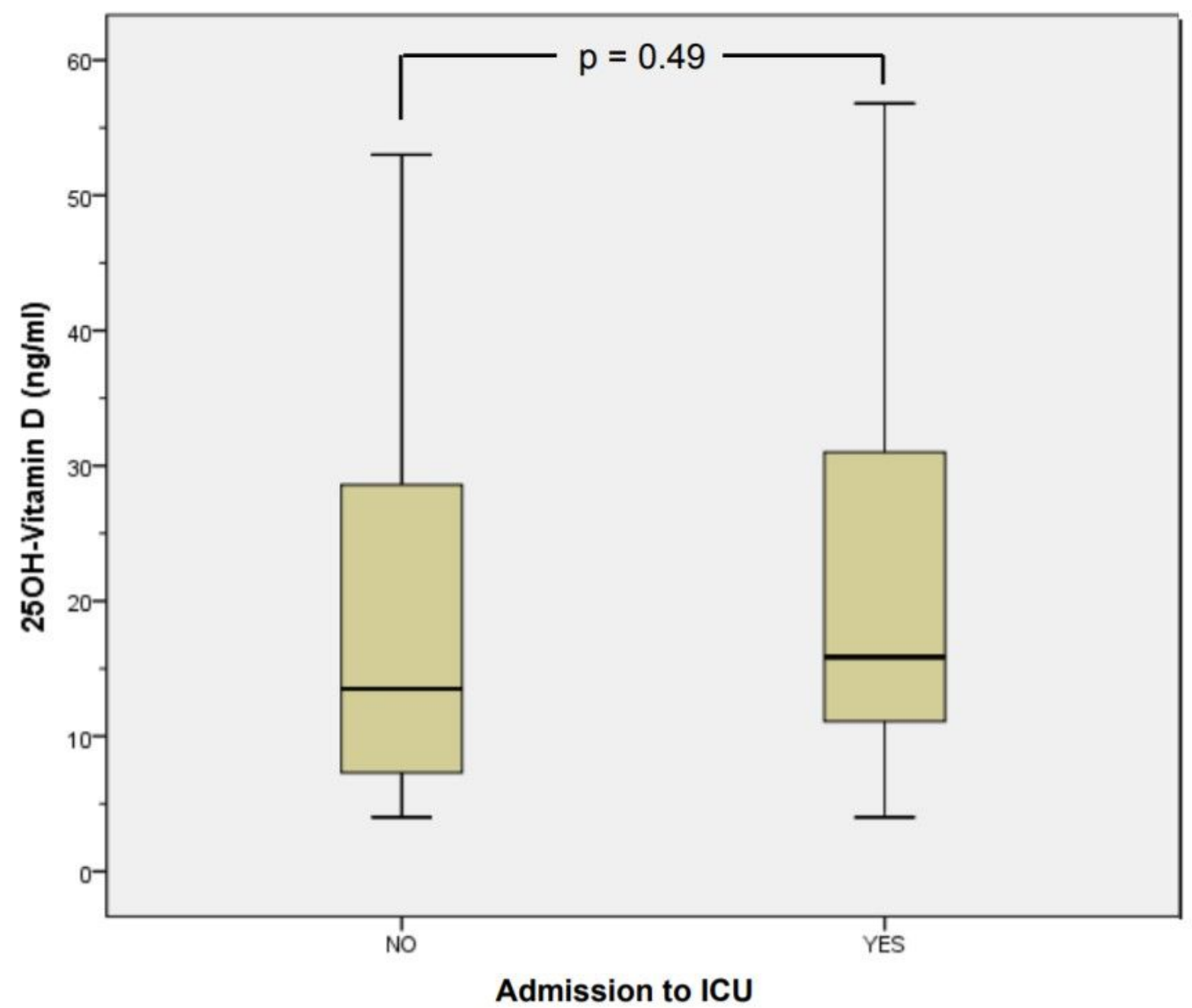

Figure 1

Comparison of the serum $250 \mathrm{H}$-vitamin $\mathrm{D}$ levels between the patients requiring mechanical ventilatory support in an Intensive Care Unit (ICU) $(n=10)$ and the patients managed in a medical ward $(n=42)$. 


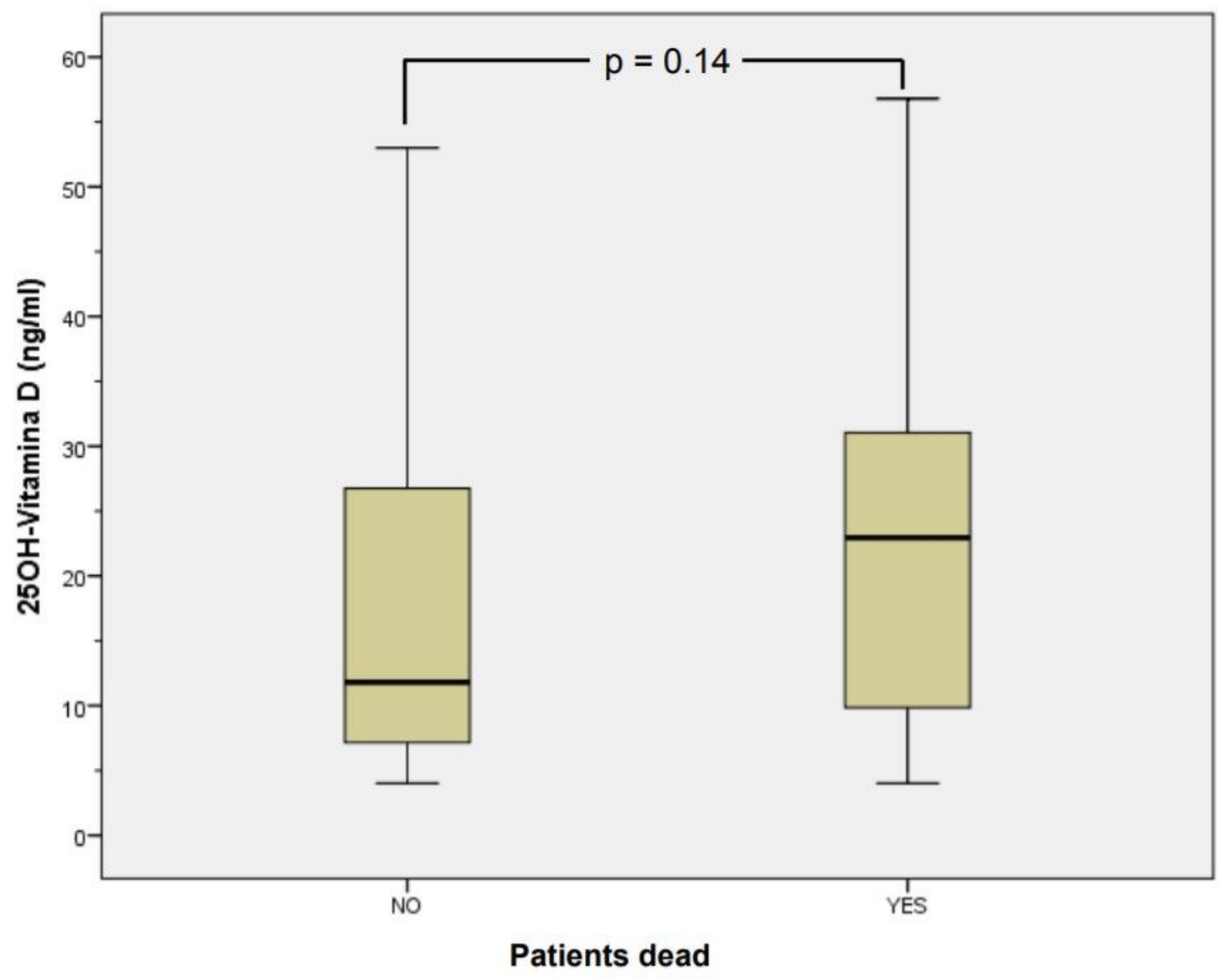

Figure 2

Comparison of the serum $250 \mathrm{H}$-vitamin D levels between the subgroup of patients died during the hospital stay $(n=16)$ and the subgroup of survivors $(n=36)$.

\section{Supplementary Files}

This is a list of supplementary files associated with this preprint. Click to download.

- Tables.pdf 